\title{
Therapeutic Effect of Micro-Ecologics Combined with Blue Light Irradiation on Neonatal Jaundice: Randomized Control Trial
}

\author{
GAO KE ${ }^{1}$, SYED HAIDER ABBAS ${ }^{1}$, ALI FAHEEM ${ }^{2}$, MUHAMMAD ZEESHAN ${ }^{2}$, RIZWAN MASUD ${ }^{2}$, MUHAMMAD AKRAM ${ }^{3}$, \\ ZAINAB AWAIS ${ }^{4}$, SYED MUHAMMAD AHSAN RAZA ${ }^{5}$, TALHA LAIQUE ${ }^{*}$ \\ ${ }^{1}$ Department of Pediatrics, The Affiliated Hospital of Ningbo university Medical College, Ningbo-China \\ ${ }^{2}$ Department of Biochemistry, CMH medical college Kharian \\ ${ }^{3}$ Department of Anaesthsiology, Major shabeer Shareef THQ level hospital, Kunjah, Pakistan \\ ${ }^{4}$ Department of Science in Dental Materials, Faryal Dental College, Lahore, Pakistan \\ ${ }^{5}$ Department of Biochemistry, M. Islam Medical and Dental College, Gujranwala, Pakistan \\ ${ }^{6}$ Department of Pharmacology, Allama Iqbal Medical College, Lahore-Pakistan \\ Correspondence to: Dr. Talha Laique, Email: talhalaique51@gmail.com Tel:+92-331-0346682.
}

\begin{abstract}
Abnormal bilirubin metabolism results in abnormally raised serum bilirubin level thus presenting as yellowing of face, skin and mucosa to varying degrees.

Purpose: To observe clinical effects on neonatal jaundice of micro-ecological preparation combined with blue light irradiation.

Study Design: Randomized control trial.

Methodology: Enrolled 100 neonatal jaundiced children (50 each group). Control group received blue light irradiation while observation group was given probiotics on the basis of blue light irradiation. Percutaneous bilirubin levels, clinical efficacy and the incidence of side effects were compared between pre and post-treatment groups. Statistical analysis: Data analyzed by SPSS $20.0 \mathrm{v}$.

Results: Post-treatment, levels of percutaneous bilirubin were significantly lower than pre-treatment. Observational group had significantly lower levels than the control group, having significant $p$-value $(P<0.05)$. However, total effective rate of the observational group was significantly higher than control group with statistically significant $p$-value $(P<0.05)$. The difference in incidence of ADR was significant $(P<0.05)$.

Conclusion: This study concluded that microecological preparation combined with blue light irradiation had a definite effect on the treatment of neonatal jaundice, not only effectively reduced the bilirubin level of children, but also reduced adverse reactions hence the safety was high. Therefore, it was worthy of application and promotion. Key Words: Neonatal Jaundice, Micro-ecological Preparations, Blue Light Exposure.
\end{abstract}

\section{INTRODUCTION:}

Abnormal bilirubin metabolism results in abnormally raised serum bilirubin level thus presenting as yellowing of face, skin and mucosa to varying degrees ${ }^{1}$. Jaundice can be either pathologic or physiologic. Pathological jaundice appear after birth $24 \mathrm{~h}$ while depict deepening phenomenon. Physiological jaundice appear 2 3 days after birth and subsides spontaneously 2 7 days later, no more than 14 days at the latest ${ }^{2}$. Pathological jaundice is extremely harmful, usually manifested as obvious yellow staining of the skin and sclera. If not treated in time, bilirubin can easily pass through the incomplete meningeal barrier, leading to the occurrence of bilirubin encephalopathy, causing irreversible damage to the central nervous system and even death ${ }^{3}$. Relevant statistics show ${ }^{4}$ that the proportion of neonatal jaundice hospitalized in China is up to $48.2 \%$, and $0.9 \%$ of these children have a more serious complication "neonatal bilirubin encephalopathy", which has a serious impact on the intelligence, motor function, visual and hearing development of the children, and is an important cause of neonatal cerebral palsy and death. Therefore, strengthening the prevention and treatment of neonatal jaundice is of great significance to prevent the occurrence of bilirubin encephalopathy. At present, there are many clinical treatments for neonatal jaundice, including blue light irradiation, immunotherapy, blood exchange therapy, antiinfection, hemolysis control and other measures, among which blue light irradiation is the most common application, but there are obvious adverse reactions, affecting its therapeutic efficacy ${ }^{5,6}$. Studies have shown ${ }^{7}$ that intestinal flora plays an important role in neonatal metabolism, and intestinal flora disorder is an important factor in inducing neonatal jaundice. Therefore, correcting intestinal flora disorder has become a new therapeutic direction. Clostridium butyricum live powder has the function of replenishing intestinal beneficial flora and promoting intestinal maturation of neonates. It is suitable for various symptoms and diseases caused by intestinal flora imbalance ${ }^{8,9}$. Currently, blue light irradiation is the main clinical treatment for neonatal jaundice. However, the use of this therapy alone in the treatment of pathological jaundice in children has significant limitations ${ }^{10}$. In this study, the clinical efficacy of micro-ecologics combined with blue light irradiation in the treatment of neonatal jaundice was reported.

Objective: To observe clinical effects on neonatal jaundice of micro-ecological preparation combined with blue light irradiation.

\section{METHODOLOGY}

Hundred neonatal jaundice children were included in present study with 50 in each group. Control group recieved blue light irradiation while observation group was given probiotics on the basis of blue light irradiation. Inclusion criteria included all children who met the relevant diagnostic criteria of neonatal hyperbilirubinemia in 
Practical Neonatology 3 and all the children were delivered at full term and had no congenital defects ${ }^{11}$. Written consent was taken and families of the children were informed about conducted study. The control group was treated with blue light irradiation, and the observation group was treated with blue light irradiation, and was treated with oral microprobiotics "Clostridium butyricum double living capsule", 420mg/ time, twice /d, and both groups were treated continuously for $72 \mathrm{~h}$. Percutaneous bilirubin levels, clinical efficacy and the incidence of adverse reactions were compared between groups pre and post-treatment.

\section{STATISTICAL ANALYSIS:}

Data analyzed by SPSS 25.0v. Measurement data were expressed as mean $\pm S D$ while $T$ or $Z$ test was used. Quantitative parameters were presented by percentage (\%). P-value $\leq 0.05$ was taken as significant.

\section{RESULTS:}

General distribution of parameters between both groups in present study was presented as mean \pm SD in Table-1.

Table-1: General Data Contrast Between Both Groups $(n=100)$

\begin{tabular}{|l|l|l|l|}
\hline Groups & Gender (male/female) & Age (days) & Birth weight (g) \\
\hline Observation group & $28 / 22$ & $3.58 \pm 1.291$ & $3.39 \pm 0.475$ \\
\hline The control group & $29 / 21$ & $3.25 \pm 1.401$ & $3.28 \pm 0.451$ \\
\hline$X^{2} / F / Z$ & 0.041 & -1.172 & -1.527 \\
\hline$P$ & 0.840 & 0.241 & 0.127 \\
\hline
\end{tabular}

Assessment of different levels of percutaneous bilirubin among enrolled subjects was presented as mean \pm SD in Table-2.

Table-2: Percutaneous Bilirubin Difference Between Both Groups ( $n=100)$

\begin{tabular}{|l|l|l|l|}
\hline Groups & Before the treatment & $\mathbf{4 8} \mathbf{~}$ after treatment & $\mathbf{7 2} \mathbf{~}$ after treatment \\
\hline Observation group & $308.0 \pm 64.147$ & $200.53 \pm 23.311$ & $65.66 \pm 13.827$ \\
\hline The control group & $294.92 \pm 75.742$ & $232.61 \pm 40.925$ & $175.28 \pm 32.226$ \\
\hline$Z$ & -1.210 & -4.283 & -8.625 \\
\hline$P$ & 0.226 & $0.000^{*}$ & $0.000^{*}$ \\
\hline
\end{tabular}

*Statistically significant

The difference in total effective rate was statistically significant between groups as shown in Table-3.

Table-3: Contrast of clinical efficacy between the two groups [N (\%)]

\begin{tabular}{|l|l|l|l|l|}
\hline Groups & Significantly effective & Effective & Invalid & Total effective \\
\hline Observation group & $37(74.00 \%)$ & $9(18.00 \%)$ & $4(8.00 \%)$ & $46(92.00 \%)$ \\
\hline The control group & $26(52.00 \%)$ & $12(24.00 \%)$ & $12(24.00 \%)$ & $38(76.00 \%)$ \\
\hline$X^{2}$ & & & & 4.762 \\
\hline$P$ & & & & $0.029^{*}$ \\
\hline
\end{tabular}

*Statistically significant

The difference in incidence of ADRs was statistically significant between groups was statistically significant as shown in Table-4.

Table-4: Contrast of side effects between the two groups [N (\%)]

\begin{tabular}{|l|l|l|l|l|l|}
\hline Groups & Fever & The Rash & Diarrhea & Dehydration & Summation \\
\hline Observation group & $2(4.00 \%)$ & $1(2.00 \%)$ & $1(2.00 \%)$ & $1(2.00 \%)$ & $5(10.00 \%)$ \\
\hline The control group & $6(12.00 \%)$ & $4(8.00 \%)$ & $3(6.00 \%)$ & $1(2.00 \%)$ & $14(28.00 \%)$ \\
\hline$X^{2}$ & & & & & 5.263 \\
\hline$P$ & & & & & $0.022^{*}$ \\
\hline
\end{tabular}

*Statistically significant

\section{DISCUSSION}

The results of this study showed that Clostridium butyricum capsules combined with blue light irradiation had obvious therapeutic effect on neonatal jaundice. They can quickly reduce the serum total bilirubin level. They quickly eliminate skin sclera yellow stain, protect important organs. However, drug toxicity is small, less adverse reactions. This study is consistent with the research results of Xia Jianxin et al. ${ }^{12}$, indicating that the combination of probiotics and blue light irradiation has a positive effect and value in the treatment of neonatal jaundice. Cen Huiling et al. ${ }^{13}$ found that another microbial agent (Bacillus subtilis bicombined living particles) combined with blue light irradiation in the treatment of neonatal jaundice has also played a good effect, but so far, no other literature has compared the differences between different microbial agents combined with blue light irradiation in the treatment of neonatal jaundice. In combination with other studies ${ }^{14-18}$.

Limitations: Our limitations included small sample size, time with financial constrains and limited resources.

\section{CONCLUSION}

This study concluded that microecological preparation combined with blue light irradiation had a definite effect on the treatment of neonatal jaundice, not only effectively reduced the bilirubin level of children, but also reduced 
adverse reactions hence the safety was high. Therefore, it was worthy of application and promotion.

Author's contribution: GK \& SHA: Conceptualized the study, analyzed the data, and formulated the initial draft. AF \& MZ: Contributed to the histomorphological evaluation. RM \& MA: Contributed to the analysis of data and proofread the draft.

ZA \& SMAR: Contributed to data collection.

TL: Contributed to the proofreading the manuscript for intellectual content.

Acknowledgements: I am thankful to Allah and my colleagues who made it possible for me.

\section{REFERENCES}

1. Rathore Shubhangi,Kumar Vk Chethan,R Sharashchandra,A critical review on neonatal hyperbilirubinemia-an Ayurvedic perspective.[J] .J Ayurveda Integr Med, 2020, 11: 190-196.

2. Watson Robin L,Hyperbilirubinemia.[J] .Crit Care Nurs Clin North Am, 2009, 21: 97-120, vii.

3. Ying Qian,You Xueqin,You Jingye. The accuracy of transcutaneous bilirubin to identify hyperbilirubinemia in jaundiced neonates.[J] .J Matern Fetal Neonatal Med, 2020, undefined: 1-8.

4. Slusher Tina M,Olusanya Bolajoko O,Vreman Hendrik J et al. Treatment of neonatal jaundice with filtered sunlight in Nigerian neonates: study protocol of a non-inferiority, randomized controlled trial.[J] .Trials, 2013, 14: 446.

5. Hansen Thor Willy Ruud,Maisels M Jeffrey,Ebbesen Finn et al. Sixty years of phototherapy for neonatal jaundice - from serendipitous observation to standardized treatment and rescue for millions.[J] .J Perinatol, 2020, 40: 180-193.

6. Slusher Tina M,Vaucher Yvonne E,Management of neonatal jaundice in low- and middle-income countries.[J] .Paediatr Int Child Health, 2020, 40: 7-10.

7. Sachdeva Monica,Murki Srinivas,Oleti Tejo Pratap et al. Intermittent versus continuous phototherapy for the treatment of neonatal non-hemolytic moderate hyperbilirubinemia in infants more than 34 weeks of gestational age: a randomized controlled trial.[J] .Eur J Pediatr, 2015, 174: 177-81.

8. Ahmadipour Shokoufeh,Baharvand Parastoo,Rahmani Parisa et al. Effect of Synbiotic on the Treatment of Jaundice in Full Term Neonates: A Randomized Clinical Trial.[J] .Pediatr Gastroenterol Hepatol Nutr, 2019, 22: 453-459.

9. Deshmukh Janki,Deshmukh Mangesh,Patole

Sanjay,Probiotics for the management of neonatal hyperbilirubinemia: a systematic review of randomized controlled trials.[J] .J Matern Fetal Neonatal Med, 2019, 32: 154-163.

10. Huang $\mathrm{H}$. Analysis of the efficacy of bifidum microbium triplet living agents combined with blue light irradiation in the treatment of neonatal jaundice [J].Systems Medicine,2019,4(04):114-116.

11. Liu Canghai, Xiao Huiqiong.Wormwood Zhi yellow and probiotics combined multiple short blue light Effects of bilirubin and albumin levels on jaundice,Drug evaluation,2019,16(14):39-41.

12. Xia Jianxin, Hu Juanjuan.Analysis of Changlekang combined with blue light therapy in the treatment of neonatal jaundice [J].Chinese and foreign medicine,2015,34(26):129-130.

13. Cen Huiling, Zheng Wenya, Pan Shuling.Effect of microecologics combined with blue light irradiation on neonatal jaundice $[\mathrm{J}]$. Chinese modern drug application,2015,9(11):7-9.

14. Wang Qi, Li Yingchun, Chen Xue.Effect of short time multiple blue light therapy in the treatment of neonatal jaundice $[\mathrm{J}]$. China modern drug application,2021,15(06):106-108.

15. Tan Bing, Jiang Guofeng. Clinical effect of Yinzhihuang granule combined with smecta in the treatment of neonatal breast milk jaundice and prognosis analysis [J].Chinese Practical Medicine,2020,15(30):149-151.

16. Shen Lingling, Lai Jiaxing.Effect of Yinzhihuang granule combined with blue light irradiation on neonatal jaundice $[\mathrm{J}]$. Shenzhen Journal of Integrated Traditional Chinese and Western Medicine,2021,31(02):26-27.

17. Zhang $\mathrm{C} M$ M. Clinical effect of microecological modulator combined with blue ray combined with Yinzhihuang granule on neonatal jaundice [J].Chinese and foreign medical research,2020,18(34):163-165.

18. Min Caifen, Shi Lifeng, Hua Juan.Effect of quality nursing intervention on neonatal jaundice $[\mathrm{J}]$. Chinese and foreign medical research,2021,19(04):108-110. 